write $a$ for the number of grains which were affected by light and express $\mathrm{D} t$, as the number of developed grains at time $T$, then

$$
\frac{d \mathrm{D}}{d t}=\mathrm{K}\left(a-\mathrm{D}_{t}\right) .
$$

If we continue development for an infinitly long time we shall develop all the exposed grains and therefore we may write $a=\mathrm{D}_{\infty}$ and

$$
\frac{d \mathrm{D}}{d t}=\mathrm{K}\left(\mathrm{D}_{\infty}-\mathrm{D}_{i}\right)
$$

which integrates into

$$
\mathrm{K}={ }_{t}^{\mathrm{I}} \log \left(\frac{\mathrm{D}_{\infty}}{\mathrm{D}_{\infty}-\mathrm{D}_{i}}\right)
$$

which is the familiar equation for a reaction of the first order.

Now the relation between the density obtained on development and the exposure which was given to the plate is given by a complicated expression which for the greater portion of the range of exposures simplifies to the expression $\mathrm{D}=\gamma(\log \mathrm{E}-\log i)$.

For a given exposure $\log \mathrm{E}-\log i$ is a constant and $\mathrm{D}$ is therefore strictly proportional to $\gamma$, so that we may write the equation we have just obtained as

$$
\mathbf{K}=\frac{I}{t} \log \left(\frac{\gamma_{\infty}}{\gamma^{*}-\gamma_{t}}\right) .
$$

In this equation $\gamma$ represents the contrast shown by the developed plate and $\gamma_{\infty}$ is therefore the maximum contrast which can be obtained on any plate with the most prolonged development. This value, $\gamma_{\infty}$, is independent of the developer employed and is a natural constant of the plate, of great importance as conditioning its behavior and property.

In concluding this brief review of the physical chemistry of development, I should like to call attention to the many interesting problems presented by the subject and to hope that this paper may induce some workers to turn to photographic chemistry as a field for their investigations.

Eastman Kopak Co.

Rochester, N. Y,

[Contributions from the Research Laboratory of Physical Chemistry of the Massachusetts Institute of Technology, No. 96.]

\title{
THE ISOLATION AND THE PROPERTIES OF SOME ELECTRO- POSITIVE GROUPS AND THEIR BEARING ON THE PROBLEM OF THE METALLIC STATE.
}

\footnotetext{
By Charies A. Kraus

Received Septembet 6, 1913.
}

The results set forth in this paper were obtained some ten years ago, being one of a number of investigations directed toward furnishing evidence as to the molecular constitution of metallic substances. At the 
time, the writer expected to be able to fill out the gaps remaining in the investigation of the electropositive groups, but circumstances intervened to interrupt the work. It seems worth while to publish the results obtained, the more so, since a number of important conclusions may be drawn therefrom.

The advance made during the past decade in our conceptions of the ultimate structure of matter, has served to focus the interest of many investigators, both chemists and physicists, on the problem of the metallic state. As yet, we are dependent upon indirect evidence for a knowledge of the nature of metallic substances, but, to one who has followed the problem closely, it seems that the time is not far distant when we may expect great advances in this heretofore inaccessible field. Considering how much we are dependent upon a knowledge of the metals for countless practical purposes, and how vitally they enter into our most fundamental conceptions of matter and electricity, we cannot doubt but that the final solution of the problem of the metallic state will mark a new epoch in scientific development.

From a chemical standpoint, the most characteristic property of metals is their power of combining with electronegative elements to form salts, or of functioning as positive ions when in solution. This property is particularly prominent in the strongly electropositive elements such as the alkali metals.

Certain groups of elements, of which none is itself metallic, possess the property of forming salts in every way similar to salts of strongly electropositive elements. Of these groups, the most familiar, and in many respects the most striking, is the ammonium group, in which four hydrogen atoms are associated with a single atom of nitrogen. The metallic nature of this group, as exemplified in its chemical properties, was recognized at a very early date; indeed, it owes its name to the clear recognition of this fact by Sir Humphrey Davy.

Many attempts have been made to isolate the ammonium group in order to determin whether or not the analogy could be extended so far as to include a free ammonium group possessing metallic properties. Thus far, all efforts to obtain the free group have met with negative results. It has been shown, however, that the ammonium group is capable of forming an amalgam whose properties are similar to those of amalgams of strongly electropositive metals.

Recently McCoy and Moore ${ }^{1}$ have studied amalgams of the substituted ammoniums. These groups are much more stable than the ammonium group itself, and the above-mentioned investigators were able to obtain amalgams in the form of crystallin metallic substances, possessing a high degree of stability at low temperatures. In their properties,

1 This Journal, 33, 273 (I9II). 
these amalgams approach very closely to amalgams of potassium and rubidium. While these experiments do not necessarily prove that the free groups are metals, they nevertheless render it highly probable that, if they could be obtained in a free state, these groups would exhibit metallic properties.

In this connection, the work of Palmaer, ${ }^{1}$ on the electrolysis of substituted ammonium salts in liquid ammonia solution, is of great importance. He found that, on electrolyzing solutions of tetramethyl and tetraethyl ammonium salts in ammonia, a blue-colored solution is formed at the cathode. This solution appears identical in color with solutions of the alkali metals, which may be formed by dissolving these metals in liquid ammonia. The writer has repeated and confirmed the experiments of Palmaer. Even tetrapropyl ammonium salts yield a blue-colored solution at the cathode. The solutions obtained by electrolysis are unstable, and the blue color disappears at the end of a few minutes.

\section{Other Electropositive Groups.}

Failing in an attempt to isolate the ammonium group, the writer undertook to investigate a number of other groups in the hope that, among them, one would be found possessing the degree of stability necessary to make its isolation possible. Salts, usually iodides or chlorides, were prepared and electrolyzed in liquid ammonia solutions at temperatures as low as $-60^{\circ}$. Ammonia not only makes it possible to carry out the electrolysis at temperatures favorable to stability, but it also provides a solvent which, even with the most electropositive metals, reacts at a very slow rate.

While the ammonium group, $\mathrm{NH}_{4}$, containing nitrogen, is the most familiar example of an electropositive group, many other elementsboth metallic and non-metallic-form similar groups with other elements and the salts of these are, in some cases, extremely stable. In most cases, however, the hydrogen derivatives cannot be obtained, although the corresponding organic derivatives are highly stable and are readily made. Only the organic derivatives were examined in this investigation.

The following salts were electrolyzed: $\left(\mathrm{CH}_{3}\right)_{4} \mathrm{SbI},\left(\mathrm{CH}_{3}\right)_{3} \mathrm{SI},\left(\mathrm{C}_{6} \mathrm{H}_{5}\right)_{2} \mathrm{II}$, $\mathrm{CH}_{3} \mathrm{HgCl}, \mathrm{C}_{2} \mathrm{H}_{5} \mathrm{HgCl}, \mathrm{C}_{3} \mathrm{H}_{7} \mathrm{HgCl}, \mathrm{C}_{5} \mathrm{H}_{11} \mathrm{HgI}, \mathrm{C}_{8} \mathrm{H}_{17} \mathrm{HgI}, \mathrm{C}_{6} \mathrm{H}_{5} \mathrm{HgI}$, and $\left(\mathrm{CH}_{3}\right)_{3} \mathrm{SnI}$. Among these substances, only the mercury alkyl salts yielded positive results. Even in the case of the alkyl mercury salts, members of the series above $\mathrm{C}_{4} \mathrm{H}_{9} \mathrm{Hg}$ did not possess sufficient stability to make their isolation possible.

Before proceeding to a discussion of the results obtained with the alkyl mercury compounds, a few remarks may be made relative to the other groups. That the group $\mathrm{Sb}\left(\mathrm{CH}_{3}\right)_{4}$ should prove unstable was to have been expected, since stibine and the stibonium compounds are much

${ }^{1}$ Z. Electrochem., 8, 729 (1902). 
less stable than ammonia and the corresponding ammonium salts. The same is true of compounds of phosphorus, arsenic, and likewise sulphur and iodine. On the other hand, the trialkyl-tin salts are extremely stable and the lack of stability of the free group is in some aspects unexpected. This behavior may, however, be due to the large number of radicals grouped around the atom of tin. In the case of the mercury group the stability decreases rapidly with increasing complexity of the attending organic radicals.

\section{Isolation of the Alkyl Mercury Group.}

The alkyl mercury salts are relatively fairly soluble in liquid ammonia. In most cases the chlorides were electrolyzed, although the nitrate was also employed. The nitrate is very soluble. Care must be exercised in handling it, since it produces painful burns when it comes in contact with the skin, the burns developing only some hours after application has taken place. It is scarcely necessary to caution the prospective investigator regarding the highly poisonous qualities of the mercury dialkyl compounds, which are quite volatil, and which are formed when the free group $\mathrm{C}_{n} \mathrm{H}_{2 n}+{ }_{1} \mathrm{Hg}$ decomposes.

Since the particular contrivance employed in carrying out the electrolyses may be varied in many ways, it is unnecessary to describe the details of the apparatus employed in this investigation. The electrolytic cell was contained in a bath of liquid ammonia, cooled below its normal boiling point in order to prevent the boiling of the solution in the cell in consequence of heat introduced by the electrolyzing current. Small platinum wires served as electrodes, the cathode being situated at the bottom of the cell.

The free group was deposited at the cathode in the form of a highly attenuated opaque mass, without evolution of gas or the production of appreciable heat. Since the precipitate is an excellent conductor, it extended itself rapidly upward through the less highly conducting solution as electrolysis proceeded. From time to time it was necessary to interrupt the current in order to compress the deposit into a more compact mass in the bottom of the cell, a suitable device being provided for this purpose. When the electrolysis had proceeded for some time, bubbles of gas began to be evolved on the precipitate and it was necessary to remove the solution and introduce a fresh one, since the bubbles tend to float the deposited metal to the top of the solution. These bubbles were doubtless hydrogen, which was evolved by the current from the reaction products formed at the anode. The solution was renewed at intervals until a sufficient quantity of the free group had been deposited at the cathode. Naturally, all manipulations were carried on under exclusion of moisture from the atmosphere. 


\section{Properties of the Alkyl Mercury Groups.}

It has already been mentioned that the group is precipitated at the cathode in an extremely attenuated form. The appearance of the deposit is black, like that of any finely subdivided metal. That it is an excellent metallic conductor is at once evident from the fact that the deposit always takes place at the top of that already formed. Since the area of the platinum wire cathode was only a few square millimeters, the current density must have been quite high in this attenuated deposit, particularly at the point of contact. with platinum wire. In no case has the deposit been precipitated in a dense form. By means of slight pressure the deposit may be brought into a fairly coherent form. In the case of the methyl mercury group, however, the deposit could not be obtained otherwise than as a black mass. None of the alkyl mercury groups amalgamate with mercury to a marked extent.

In order to test some of the properties of the free group, a quantity of the metal $\left(\mathrm{CH}_{3} \mathrm{Hg}\right)$ was prepared as described above. The product was freed from salt by repeated washing with pure ammonia. The last traces of ammonia were removed by means of a pump, the tube being immersed in a bath of ammonia at its boiling point. The tube was then bent into the form of an inverted $U$ and sealed off under an internal pressure of about a millimeter.

On allowing the mass to warm up, the surface became gray, owing to decomposition of the compound at the surface. The whole mass, however, did not decompose until very near ordinary temperatures, when decomposition took place suddenly under evolution of considerable heat. Immediately on decomposition, the contents of the tube separated into metallic mercury and a clear liquid which was afterward shown to be mercury dimethyl. The other arm of the $\Omega$-tube was now cooled off in liquid ammonia, while the first arm was slightly warmed. The clear liquid distilled over, leaving the metallic mercury behind. The products were weighed separately and gave 48 per cent. of metallic mercury. The percentage of mercury found is thus somewhat high. (Assuming decomposition to take place according to the equation, $2 \mathrm{HgCH}_{3}=\mathrm{Hg}+\mathrm{Hg}$ $\left(\mathrm{CH}_{3}\right)_{2}$, the result should have been 46.6 per cent.) This result is explained by the fact that the compound had already partially decomposed, the mercury remaining behind, while the mercury dimethyl was washed away. That there is appreciable decomposition at $-33^{\circ}$, was shown by allowing a quantity of the material to stand over night in a bath of ammonia. A portion, though less than half, was decomposed. As the electrolysis required three or four hours, it is evident that the amount of mercury found must necessarily have been too high.

To establish the nature of the clear liquid which results when the free group decomposes, it was treated with acid. Under these conditions, 
methyl mercuric salts were obtained, showing conclusively that the liquid is $\mathrm{Hg}\left(\mathrm{CH}_{3}\right)_{2}$.

That ammonia is not associated with the cathode product is shown by the resulting pressure after decomposition, as well as by the fact that the same compound may be obtained in other solvents than ammonia. In water solution, little black flakes form at the cathode but soon disappear. In alcohol solution, however, a considerable deposit may be obtained, particularly at lower temperatures. That the body is a compound and not a mixture of some kind is evident from the manner in which it decomposes, as well as from the fact that considerable heat accompanies this decomposition.

Methyl, ethyl, propyl, amyl, and octyl derivatives of mercury were studied. The octyl derivative gave no indication of being able to exist free. The amyl derivative gave slight indication of coming down at the cathode, but its decomposition followed almost immediately. Between the methyl and ethyl derivatives, little difference could be observed in stability, but the propyl derivative appeared to be decidedly less stable. It is probable that the butyl derivative may be obtained.

There is a very striking change in the nature of the cathode product as the higher members of the séries are approached. As already stated, the methyl mercury group comes down as a black deposit which could not be obtained in any other form. Ethyl mercury comes down in the same way as a black deposit, but when it is compressed slightly, it coheres and exhibits metallic reflection, somewhat of a copper color. Even on allowing a quantity of this substance to stand some time, it coheres and gives a metallic reflecting surface on the walls of the tube. When electrolyzed on a mercury cathode, the same color is observed at the beginning. From this behavior of methyl and ethyl derivatives, it was thought probable that propyl mercury would exhibit metallic lustre in even a more marked degree, and such was indeed found to be the case. Even in the loose, spongy form in which it comes down, it exhibits a decided reflecting power similar to that of the ethyl mercury group described above. It is probable that these differences as to lustre are largely due to the fact that the higher derivatives are softer.

When methyl mercury warms up, it decomposes without melting at approximately room temperatures. In the case of ethyl mercury, the compound melts right on the point of decomposition. There seems to be a small quantity of reddish colored liquid formed, the color of which diasppears almost immediately. The circumstances attending the formation of the reddish colored liquid have not been studied further. It is probable that propyl mercury, when prepared in quantity, will show the same behavior in a more pronounced manner. 


\section{Discussion.}

There can be no question that the free groups: $\mathrm{CH}_{3} \mathrm{Hg}, \mathrm{C}_{2} \mathrm{H}_{5} \mathrm{Hg}$, and $\mathrm{C}_{3} \mathrm{H}_{7} \mathrm{Hg}$ have been obtained in a free state and that, in this state, they possess metallic properties, or, for short, are metals. While this result possesses much interest from a purely chemical standpoint, its chief importance lies in the light it sheds on the nature of metallic substances and the nature of the process of metallic conduction.

It is unfortunate that these groups are unstable; for it would be of great interest to study the influence of chemical constitution on the optical and electrical constants of these metals. Nevertheless, we may draw certain important conclusions from the mere fact that these compounds are metallic in nature.

According to the electron hypothesis, the peculiar characteristics of metallic matter are due to the presence of one or more molecular species common to all metals, each species being associated with a definit charge of electricity. This hypothesis is well substantiated by many facts, particularly by the absence of any manner of material effects when a current passes through a metal or through the boundary between two metals.

Two further assumptions are usually made; the first, that only a single species of carriers exists in a metal, namely: the negatively charged carrier; and second, that the mass of this carrier is approximately $\mathrm{r} / 1000$ that of the hydrogen atom. These last two assumptions constitute extrapolations based on our knowledge of the conduction process in gases and in electrolytes. They thus rest on a much weaker foundation than the first hypothesis which is based on direct observations on the metals themselves. The greatest uncertainty exists, as to whether more than one molecular species is present and takes part in the conduction process in metals. In the theoretical treatment of the subject many physicists are wont to take more than one species into account.

Assuming that only one species is concerned in the conduction process, direct evidence is lacking as to whether this species is negatively or positively charged. The presumption is that it is negatively charged, since in gases only negatively charged carriers are known, whose mass is suffciently small to readily account for the high conductance of metals. So far, however, evidence obtained from a study of metallic systems themselves is inconclusive. An attempt will be made to furnish this evidence below.

An important result gained from this investigation into the nature of the electropositive groups is that the metallic state is a molecular and not an atomic property of matter.

This conclusion is not based entirely on the results obtained by the writer with the alkyl metcury groups and those obtained by others with 
amalgams of the ammonium and the substituted ammonium groups, although, taken together they present strong evidence in its support.

In an extended investigation, the results of which remain in part unpublished at this time, the writer has demonstrated the metallic nature of the blue-colored solutions obtained by dissolving the alkali metals in liquid ammonia ${ }^{1}$. The solutions obtained by Palmaer, by electrolyzing solutions of substituted ammonium salts in liquid ammonia, exhibit the same characteristic blue color as do solutions of the alkali metals. There can be no question that the solutions of the free groups $\left(\mathrm{C}_{n} \mathrm{H}_{2 n}+1\right)_{4} \mathrm{~N}$ are identical in nature with solutions of the alkali metals, and that, therefore, this group, which is made up entirely of non-metallic elements, is itself a metal.

If the metallic state is a molecular and not an atomic property of matter, we may inquire: What constitutional relationship does exist among metals? In answer to this question we may say that all metals possess in common the tendency to form positive ions, and the greater the tenddency to form positive ions, i. e., the more electropositive a metal is, the more accentuated are its distinctively metallic characteristics. The alkali metals are thus metals which in their physical properties exhibit the characteristics of metals in the most marked degree. Not only is the conducting power of the alkali metals very high, but their reflecting power is enormously greater than that of any other class of metallic substances. Thus, the amount of unreflected light from surfaces of mercury, platinum, silver, and sodium is, respectively; $21.6,30,5.0$, and 0.3 per cent.

If then, there is a relation between the electropositive nature of an element or group of elements and the metallic nature of the free element or group of elements, and if the metallic state is due to the presence in the element or group of elements of charged carriers free to move, it follows that these carriers must be either positively or negatively charged, but both positively and negatively charged carriers cannot be present at the same time. For, if we separate the electropositive from the electronegative constituent (as by electrolysis) of a non-metallic compound, made up of a non-metallic electronegative element and an electropositive group of non-metallic elements, we find that the electropositive constituent is metallic and the electronegative constituent non-metallic. In the separation, therefore, the electropositive group has either gained a freely movable negative carrier or lost a positive carrier. Bearing in mind the fact that the metallic state is in no wise a property characteristic of elements which are themselves metallic, we may definitly say that the electropositive group has gained a freely movable negative carrier. For, had the electropositive group lost a positive carrier in the process of separation, then, in the compound, there would be more carriers

1 Trans. Am. Electrochem. Soc., 21, I19 (Igr2). 
associated with the metallic constituent than in the free group, and the compound would necessarily possess metallic properties; since, in the addition of carriers to a group in which there are already carriers of the same kind possessing freedom of motion, the added carriers must possess a freedom of motion at least as great as that of those originally present. Since, however, the compound is non-metallic, and therefore there are no carriers present possessing freedom of motion, we must conclude that, in the compound, the electropositive group has lost a freely movable negative carrier. In the process of combination with an electronegative constituent, therefore, the freedom of motion of the negative carrier is lost, the carrier being bound to the negative constituent. When the positive and negative constituents are separated, the positive group regains its movable negative carrier and consequently its metallic properties.

To make the argument conclusive, we must consider two points somewhat further. First, it may be contended that, when combination takes place between the positive and negative constituents, something in the nature of a high frictional resistance is introduced to the motion of the carrier, owing to the presence of essentially non-metallic elements. That such is not the case is conclusively shown by the fact that the compound $\mathrm{CH}_{3} \mathrm{Hg}$, for example, contains non-metallic elements and yet is an excellent metallic conductor. Further, the compound $\left(\mathrm{CH}_{3}\right)_{2} \mathrm{Hg}$ is a nonmetal, while the compound $\mathrm{C}_{3} \mathrm{H}_{7} \mathrm{Hg}$, containing a greater number of both carbon and hydrogen atoms, is a metallic conductor. So too, a solution of tellurium in liquid sulphur is a metallic conductor, as is also a solution of sodium in liquid ammonia. Similarly, the solid compound $\mathrm{Ca}\left(\mathrm{NH}_{3}\right)_{6}{ }^{1}$ is a metallic conductor. We conclude, therefore, that a nonconductor is not one in which great resistance exists to the motion of the charged carriers characteristic of metallic conductors, but one in which there are present no carriers free to move. If, by any means whatsoever, we introduce into a non-metallic substance uncombined negative electrons, the resulting system will be metallic. We may do this by dissolving a metal in a non-metallic medium as, for example, by dissolving sodium in ammonia; or we may do it by combining with a non-metallic element other elements which either furnish the negative electron themselves, or which so alter the force acting between the non-metallic element and its electron as to permit dissociation between the two, as is the case in the ammonium group.

The second objection, which may be urged against the conclusion that only the negative carriers conduct the current in metal, is that the power of conducting the current may be an intrinsic property of metallic atoms. This, however, is completely met by the fact that an electropositive group, containing no metallic elements is a metal. It has, therefore,

THe JorkN.31. 30, 665 (1908) 
been shown that, in a metal, the current is carried by negatively charged carriers.

There has been much uncertainty among physicists as to the source of the negative carriers in metals. The results obtained in the study of radioactive substances have shown that atoms are very complex systems containing a considerable number of electrons. It has not always been clear as to what electrons in the atom are concerned in the conduction process. As a result of the study of electropositive groups, it is clear that it is necessary to distinguish between two classes of electrons in metals. There are certain electrons which enter into the internal structure of the atom itself. These are practically fixed in their orbital relations, and, possessing enormous energy, they are entirely unaffected by any change in the conditions to which we are at the present time able to subject them. On the other hand, in metals there are also present electrons which are held but loosely, which are in fact able to move freely from atom to atom. These electrons are very sensitive to changes in condition such as temperature, pressure, the presence of other atoms, etc. So weak is the bond uniting the electron to an atom that more electronegative atoms may remove it entirely from the original atom in question. The electrons to which conduction is due in metals are the same electrons which are involved in the common chemical combination of metals with other elements.

In chemical combinations the electronegative constituent takes up an additional negative electron for which the positive constituent has but a very small attraction. The less tendency the metal has to retain its electron, the more electropositive it becomes and the more readily does it, in general, react. Ordinarily, the positive and negative constituent. of a compound are held together through the medium of the electrons Under certain conditions, however, (for example in solution in a dielectric medium) the electrostatic force acting between the metallic atom and its electron becomes weakened to such an extent that the negative constituent escapes carrying the electron with it. The same result may be obtained at high temperatures with the fused salt or even with the solid compound.

Boston, Mass.

[CONTRibution from the Chemical Lagoratory of the Orggon Agricultural, EXPERIMENT StATION.]

\section{ON THE REACTION BETWEEN SULFUR AND POTASSIUM HY- DROXIDE IN AQUEOUS SOLUTION. \\ By HeRMaN V. TARTAR. \\ Received August 11, 1913.}

Introduction.

In connection with some investigations on the "lime-sulfur" spray, now so widely used as an insecticide, the author had occasion to look up 